\title{
Intracranial extension of choroidal melanoma via the optic nerve*
}

\author{
C LALLY SHIELDS, ' JERRY A SHIELDS, ' DAVID L YARIAN," \\ AND JAMES J AUGSBURGER'
}

From the 'Oncology Service, Wills Eye Hospital, Jefferson Medical College, Thomas Jefferson University, Philadelphia, and the ${ }^{2}$ Retina-Vitreous Center, East Brunswick, NJ, USA

SUMMARY A 62-year-old woman was treated with cyclocryotherapy for neovascular glaucoma of uncertain origin. Fifteen months later she was diagnosed as having a choroidal melanoma, which extended extrasclerally to fill the orbit and invaded the optic nerve intracranially to the chiasm. She was treated by orbital exenteration and neurosurgical resection of the intracranial portion of the optic nerve. The patient is alive and clinically free of metastasis five years after the onset of neovascular glaucoma and almost four years after surgical resection. The significance of this highly unusual growth pattern of uveal melanoma is discussed.

Invasion of the optic nerve by uveal melanoma is rare.' In a histopathological review of 2535 cases of uveal melanoma Wilder and $\mathrm{Paul}^{2}$ reported that only $0.6 \%$ of choroidal and ciliary body melanomas invade the optic nerve. Microscopically the majority of juxtapapillary uveal melanomas stop abruptly at the optic disc margin and do not extend into the axial portion of the optic nerve. We report a case of choroidal melanoma with massive orbital invasion and extensive optic nerve infiltration to the optic chiasm.

\section{Case report}

A 62-year old white woman with no prior medical or ocular problems noted soreness of her left upper eyelid in December 1980. She was diagnosed as having neovascular glaucoma and a dense cataract in the left eye. Ophthalmoscopy of the involved eye was limited because of the lens opacity. The patient was treated with topical corticosteroids, atropine, and two courses of cyclocryotherapy, but her intraocular pressure remained elevated. B scan ultrasonography of the left eye revealed a retinal detachment with

*Presented at the Midwestern Ophthalmic Pathology Society (Theobald Society) Meeting, New Orleans on I 6-7 March, 1986.

Correspondence to Jerry A Shields, MD, Oncology Service, Wills Eye Hospital, 9th and Walnut Streets, Philadelphia, PA 19107, USA. either tumour or subretinal blood. The patient declined further examination and was followed up at periodic intervals for glaucoma and pain control.

She noted a gradual onset of a 'bulging' left eye in March 1982 and was found to have proptosis, limited extraocular motility of the left eye, and an orbital mass. She was referred to the Wills Eye Hospital Ocular Oncology Service for further treatment.

Ocular examination revealed a visual acuity of $6 / 6$ $(20 / 20)$ in the right eye and no light perception in the left eye with a Marcus Gunn pupil. Examination of the right eye revealed no abnormal findings. Hertel exophthalmometry demonstrated $14 \mathrm{~mm}$ of left proptosis. Ductions of the left eye were severely limited in all fields of gaze, and external examination revealed a $4 \times 3 \times 3 \mathrm{~mm}$ firm subcutaneous mass inferior to and including the left lower eyelid (Fig. 1). Biomicroscopy of the left eye showed early band keratopathy, shallow anterior chamber, diffuse iris atrophy, and a dense cataract displacing the iris anteriorly (Fig. 2). Intraocular pressures were 16 $\mathrm{mmHg}$ in the right eye and $64 \mathrm{mmHg}$ in the left eye, and gonioscopy of the left eye revealed a completely closed angle. The dense cataract precluded an ophthalmoscopic view of the fundus.

B scan ultrasonography and computed tomography (CT) of the orbits showed a large mass filling the globe and entire orbit (Fig. 3). A Goldmann visual field of the right eye did not reveal any defects suggesting chiasmal or right optic nerve involvement.

A fine needle aspiration biopsy of the inferior 


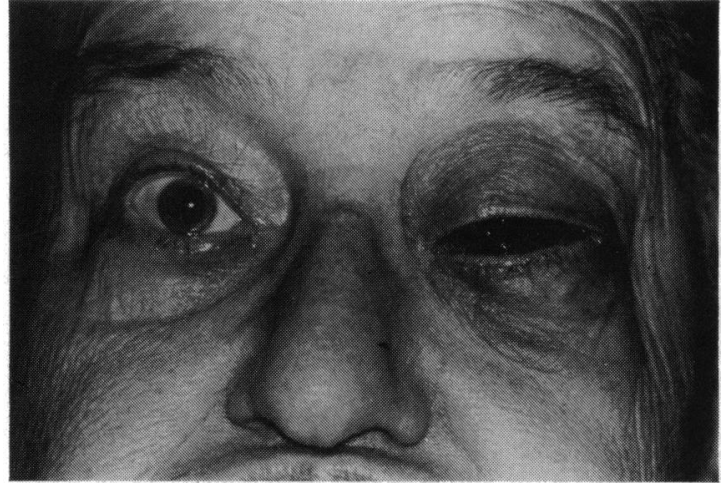

Fig. 1 External photograph of face showing left proptosis, periorbital oedema, and conjunctival injection.

orbital mass revealed malignant cells (class V) compatible with malignant melanoma (Fig. 4). A thorough systemic examination with liver enzymes, chest $x$-ray, and liver CT showed no evidence of distant metastases.

The patient's left orbit was exenterated with a lid sparing technique. At the time of surgery the transected optic nerve near the orbital apex was found to be black. Further resection of the optic nerve to the posterior portion of the optic canal continued to show black infiltration of neural tissue into the optic foramen. Because of the extension of the tumour into the optic canal the patient was referred for neurosurgical evaluation. It was decided to resect the prechiasmal portion of the left optic nerve. Postoperatively the chiasm was irradiated with a total fractionated dose of $6000 \mathrm{rad}$. (60 Gy).

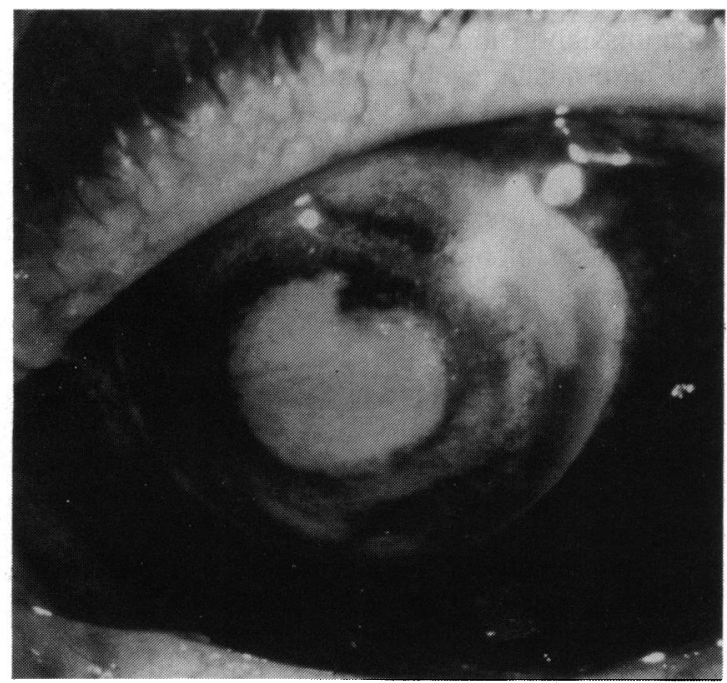

Fig. 2 External photograph of left eye showing a dense cataract displacing the iris lens diaphragm anteriorly.

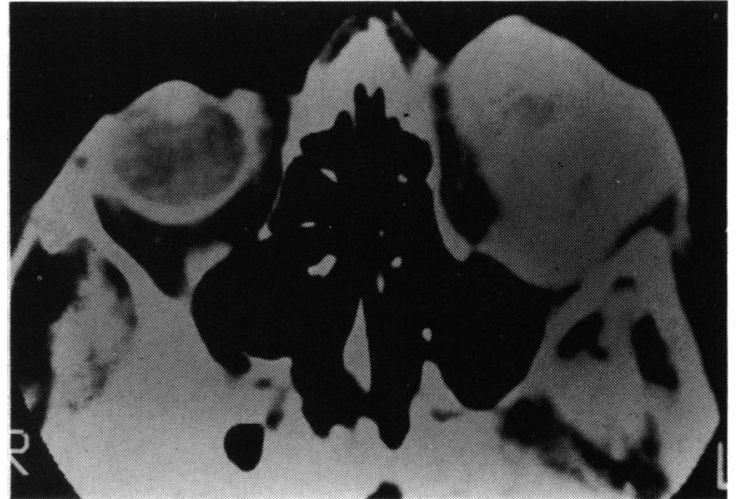

Fig. 3 Computed tomography of the orbits showing the left eye filled with a dense mass with extensive orbital involvement.

The patient had no further problems until December 1984, when she developed an enlarged preauricular node, which was excised and found to contain malignant melanoma cells. A further search for metastases was negative, and the patient was treated with local radiotherapy to the left preauricular and cervical lymph nodes.

The patient is alive, well, and free of distant metastases five years after her initial presentation.

\section{PATHOLOGY}

The exenteration specimen consisted of the eyelids, globe, and periocular orbital tissue. Gross examination of the sectioned specimen showed a globe filled with a light brownish grey mass pushing the opaque lens forward and flattening the anterior chamber. A multilobulated, firm, tan coloured mass $(23 \times 36 \mathrm{~mm})$ emerged from the inferotemporal aspect of the globe to replace completely the periocular tissue. The optic nerve was black along its entire length, without gross evidence of dural pigmentation (Fig. 5).

Microscopic examination showed a massive tumour filling the vitreous cavity and displacing the iris-lens diaphragm anteriorly. The atrophic iris was flattened against the corneal endothelium. The lens showed dense nuclear sclerosis and liquefaction of the cortex with a ruptured capsule. The ciliary body was atrophic.

The tumour was composed predominantly of epithelioid melanoma cells (Fig. 6) with nuclear pleomorphism and numerous mitotic figures. Focal areas of tumour necrosis were present. On crosssection of the optic nerve, there was profound optic atrophy and focal aggregates of erythrocytes with melanocytic neoplastic cells infiltrating the pial columns to the site of transection. The subarachnoid space and dura mater were free of tumour cells. 
Fig. 4 Cytology of the fine needle aspiration biopsy showing large rounded malignant melanoma cells with characteristic cytoplasmic pigment granules. Papanicolaou stain.

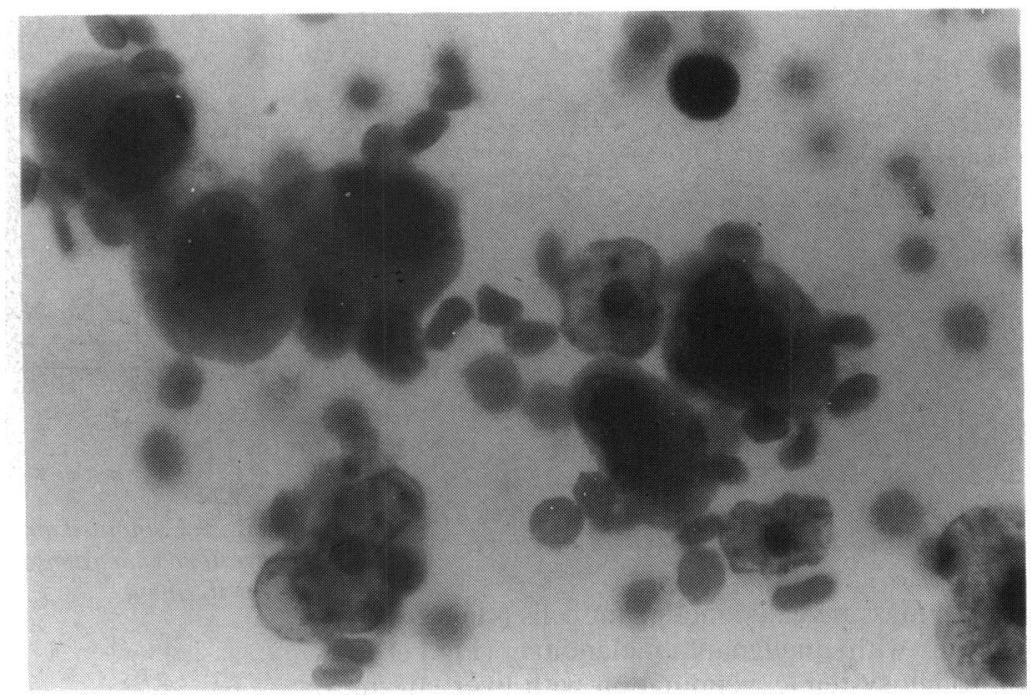

pathologically showed optic nerve extension. All the eyes had no perception of light, angle closure glaucoma, and cavernous degeneration of the optic nerve. He proposed that the mechanism of optic nerve invasion involved tumour seeding into the vitreous with subsequent invasion of the optic nerve. The cavernous spaces of glaucomatous optic atrophy have been shown to contain hyaluronic acid, presumably of vitreous origin. It is possible that the vitreous substance within the cavernous spaces carried the neoplastic cells into the nerve.

Spencer ${ }^{5}$ emphasised that absence of light perception is distinctly unusual in typical cases of uveal melanoma, but it was characteristically found in his series of melanomas with optic nerve extension. Shammas and Blodi ${ }^{3}$ reported 16 cases of peripapillary choroidal melanomas that extended into the optic nerve and 10 cases of peripapillary melanomas that were without optic nerve extension. They found that all the eyes with optic nerve extension were blind and glaucomatous and only one eye without optic nerve extension was blind or glaucomatous. Thus absence of light perception in an eye with a melanoma may suggest the presence of optic nerve invasion by the tumour.

Large tumour size ${ }^{6}$ and diffuse growth pattern ${ }^{7}$ are also factors associated with optic nerve extension. Diffuse growth pattern of choroidal melanoma is defined as tumour mass involving a minimum of onequarter of the uvea (including the iris and ciliary body) and less than $7 \mathrm{~mm}$ in height. ${ }^{8}$ This represents only $5 \%$ of all choroidal melanomas, and this tumour type is frequently misdiagnosed because of its flat appearance. ${ }^{8}$ Brown and Shields ${ }^{7}$ reported two cases of diffuse choroidal melanoma with optic disc oedema. On histopathological examination of the
Fig. 5 Gross examination of the exenteration specimen showing the globe, orbit, and optic nerve with tumour infiltration. Note the black colour of the optic nerve (arrow). 


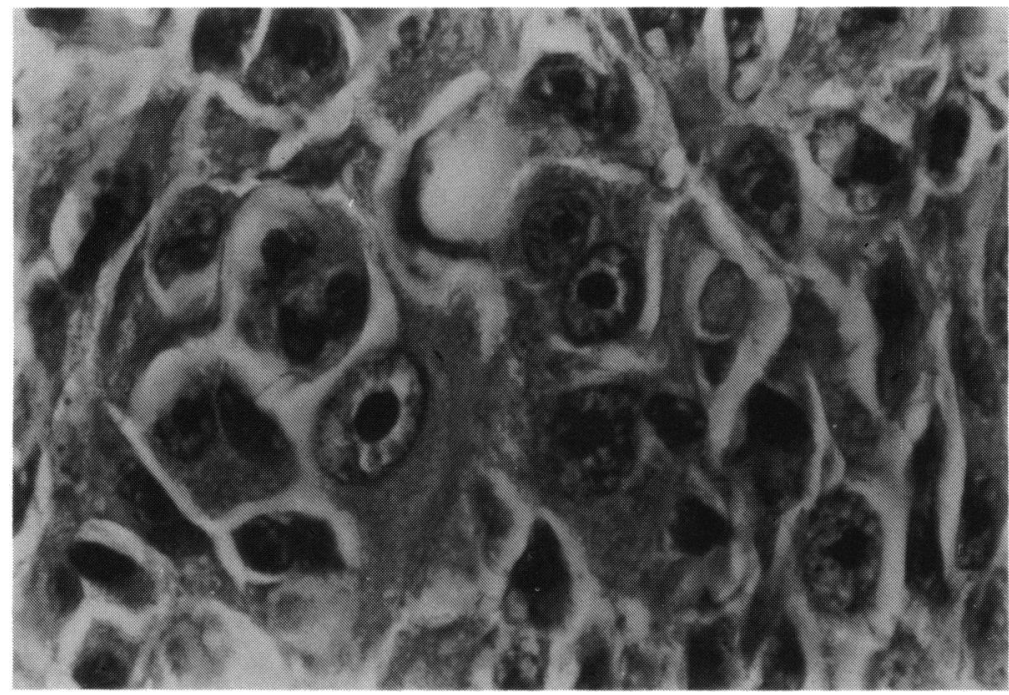

Fig. 6 Photomicrograph showing epithelioid melanoma cells. Haematoxylin-eosin.

two cases only one had optic nerve invasion. They pointed out that optic nerve invasion was characterised by a swollen yellow optic disc which was hypofluorescent on fluorescein angiography. Preoperative determination of possible optic nerve extension is important in assessing the prognosis for the patient and in planning surgery with attempts to obtain a long section of nerve at enucleation. ${ }^{45}$

Uveal melanomas are termed peripapillary when they surround the optic nerve head. In 1973 Scuderi $^{9}$ reported a case of peripapillary melanoma with optic nerve extension. Shammas and Blodi ${ }^{3}$ reported 26 cases of peripapillary melanoma and claimed $61.5 \%$ extended into the optic nerve and $46.2 \%$ extended into the subarachnoid space. Our experience at Wills Eye Hospital Oncology Service has been that nodular peripapillary melanomas have little tendency to invade the nerve, while diffuse, relatively flat peripapillary melanomas show a marked propensity for optic nerve extension.

The type of cell was epithelioid or mixed in all 10 cases of optic nerve extension of uveal melanoma reported by Spencer ${ }^{5}$ and in $87.5 \%$ of the cases of optic nerve extension reviewed by Shammas and Blodi. ${ }^{3}$ Likewise the cases reported by Chess and associates $^{6}$ and Scuderi, ${ }^{9}$ and the case we report here, were tumours composed of epithelioid cells. Within the epithelioid cell mass tumour necrosis has commonly been described. ${ }^{23} \mathrm{~s}$

Management of choroidal melanomas with optic nerve extension requires a gentle enucleation with excision of a long piece of optic nerve. ${ }^{35}$ Observation of the cut surface of the optic nerve during the surgical procedure may reveal incomplete excision of tumour by a typical black colour of the transected edge. This finding should direct the surgeon to excise a more posterior portion of the nerve. ${ }^{5}$ It is uncertain whether complete local excision improves the systemic prognosis. Spencer ${ }^{5}$ believes that the poor prognosis is related to the features associated with optic nerve invasion such as epithelioid cell type, necrosis, and large tumour size. Shammas and Blodi ${ }^{3}$ state that the prognosis of choroidal melanoma with optic nerve extension is dependent primarily on the presence of concurrent orbital invasion.

The role of exenteration and/or radiotherapy for uveal melanoma with extrascleral extension is uncertain ${ }^{130-13}$ Our patient, with massive extraocular spread, was treated with orbital exenteration, neurosurgical resection of the intracranial portion of the optic nerve, and postoperative radiotherapy. Although optic nerve extension is considered a poor prognostic sign,${ }^{14}$ our patient is healthy and without evidence of distant metastases at the time of this writing, five years after she presented with a blind painful eye.

Supported in part by the Ocular Oncology Fund and the Oncology Research Fund, Wills Eye Hospital, Philadelphia and the Black Patch Invitational Golf Tournament, Downingtown, PA.

\section{References}

1 Shields JA. Posterior uveal melanomas: clinical and pathologic features Diagnosis and management of intraocular tumours. St Louis: Mosby, 1983: 144-70.

2 Wilder HC, Paul EV. Malignant melanoma of the choroid and ciliary body: a study of 2535 cases. Milit Surg 1951; 109: 370-8.

3 Shammas HF, Blodi FC. Peripapillary choroidal melanomas. Arch Ophthalmol 1978; 96: 440-5.

4 Spencer WH. Primary neoplasms of the optic nerve and its sheaths: clinical features and current concepts of pathogenetic mechanisms. Trans Am Ophthalmol Soc 1972; 70: 490-528.

5 Spencer WH. Optic nerve extension of intraocular neoplasms. Am J Ophthalmol 1975; 80: 465-71. 
6 Chess J, Albert DM, Bellows AR, Dallow R. Uveal melanoma: case report of extension through the optic nerve to the surgical margin in the orbital apex. Br J Ophthalmol 1984; 68: 272-5.

7 Brown G, Shields JA. Mechanisms of optic disc swelling with diffuse choroidal melanomas: clinicopathologic correlations. $\mathrm{Br}$ J Ophthalmol 1982; 66: 77-82.

8 Font RL, Spaulding RG, Zimmerman LE. Diffuse malignant melanoma of the uveal tract: a clinicopathologic report of 54 cases. Ophthalmology (Rochester) 1968; 72: 877-95.

9 Scuderi G. Juxtapapillary malignant melanoma. Ophthalmologica 1973; 166: 349-52.

10 Starr HJ, Zimmerman LE. Extrascleral extension of choroidal and ciliary body melanomas. Int Ophthalmol Clin 1962; 2: 369-85.
11 Affeldt JC, Minckler DS, Azen SP, Yeh L. Prognosis in uveal melanoma with extrascleral extension. Arch Ophthalmol 1980; 98: 1975-9.

12 Shields JA, Cruess AF, Augsburger JJ, Corwin C. The management of extraocular extension of posterior uveal melanomas. Orbit 1982; 1: 209.

13 Shammas HF, Blodi FC. Orbital extension of choroidal and ciliary body melanomas. Arch Ophthalmol 1977; 95: 2002-5.

14 Weinhaus RS, Seddon JM, Albert DM, Gragoudas ES, Robinson N. Prognostic factor study of survival after enucleation for juxtapapillary melanomas. Arch Ophthalmol 1985; 103: 1673-7.

Accepted for publication 30 May 1986. 